\title{
Towards the Applied Hybrid Model in Decision Making: A Neuropsychological Diagnosis of Alzheimer's Disease Study Case
}

\author{
Ana Karoline Araújo de Castro \\ Plácido Rogério Pinheiro \\ Mirian Calíope Dantas Pinheiro \\ Isabelle Tamanini \\ Graduate Program in Applied Computer Sciences, University of Fortaleza, \\ Av. Washington Soares, 1321 - Bl J sl 30 \\ Fortaleza, Ceará 60811-905, Brazil \\ E-mail: \{akcastro, isabelle.tamanini\}@gmail.com,\{placido, caliope\}@unifor.br \\ www.unifor.br/mia
}

Accepted: 27-12-2009

Received: 19-09-2010

\begin{abstract}
A hybrid model, combining influence diagrams and a multicriteria method, is presented in order to assist with the decision making process about which questions would be more attractive to the definition of the diagnosis of Alzheimer's disease, considering the stages of Clinical Dementia Rating. The modeling and evaluation processes were carried out through a battery of standardized assessments for the evaluation of cases with Alzheimer's disease developed by Consortium to Establish a Registry for Alzheimer's disease.
\end{abstract}

Keywords: Alzheimer's disease, Diagnosis, Multicriteria Method, Bayesian Network, Influence Diagram

\section{Introduction}

With the advance of sciences applied to the health area in the last few years, there has been a considerable increase in the life expectancy of the population. Such fact can be stated based on demographic studies in developed and developing countries, which have showed a progressive and significant increase in the elderly population in the last years ${ }^{1}$. Along with this fact, a major increase in the number of health problems among the elderly can be noticed. These problems, besides being long-duration problems, require skilled personnel, a multidisciplinary team, equipment and additional high-cost tests.

Among the illnesses that occur, especially in elderly people, we can say that the dementia is the one that deserves a major attention, since the chances of presenting the pathology increase exponentially as one gets older. Dementias are syndromes characterized by a decline in memory and other neuropsychological changes. Such situation of impairment can be described by three main characteristics:

- loss of skills acquired throughout life, such as: driving, getting dressed, cooking, etc;

- loss of memory, ranging from a simple oversight to a more severe case such as not remembering one's own identity; and

- behavior problems such as agitation, insomnia, tearfulness, inappropriate behavior, loss of normal social inhibition, etc.

The Alzheimer's disease is the most frequent cause of dementia and is responsible (alone or in association with other diseases) for $50 \%$ of the cases in western countries ${ }^{1}$. 
The disease was also recognized as the fifth leading cause of death in 2003 among people older than 65 years old. Besides, its incidence and prevalence double every 5 years, with estimated prevalence of $40 \%$ among people with more than 85 years of age ${ }^{2}$.

In spite of its high incidence, doctors fail to detect dementia in 21 to $72 \%$ of their patients ${ }^{3}$ and studies have shown that the pathology has already been present for decades when the diagnosis is made.

The Alzheimer's disease (AD) is a progressive and degenerative disease of the brain which causes serious impairments over its two main activities: thinking and memory. It is a difficult diagnosed illness: the initial symptoms are subtle, progressing slowly until they are clear and devastating. The brain changes caused by the disease are shown in fig 1.

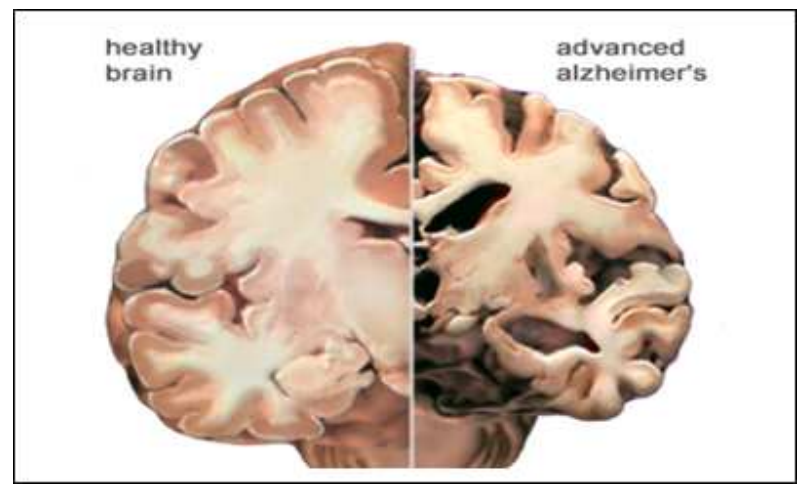

Fig. 1. Differences between a normal and an AD brain.

The accuracy of the diagnosis is very important, and each day more treatments become available ${ }^{4}$.

Due to treatment limitations at late stages of the disease, the early diagnosis is of great importance, because it improves the quality of life of patients and their families. Besides, the earliest the dementia is diagnosed, the greater the chances of delaying its advancement. This way, the major achievement of the researches nowadays would be to find a way to identify the disease in its earliest stages.

One way to identify whether a patient is having normal aging or is developing some form of dementia, is through a neuropsychological evaluation ${ }^{3}$. There are several tests available, and one of the major challenges is to find out which test would be more efficient to establish the diagnosis of dementia. One factor that must be observed is the brevity of the test, that is, the shorter the test is, the more effective it will be.
The main focus of this work is to develop a hybrid model, combining influence diagrams and multicriteria methods, for aiding on the establishment of which questions are the most attractive, considering the stages of CDR (Clinical Dementia Rating), in decision making for the diagnosis of Alzheimer's disease. The information needed to do this is based on the data collected from a battery of tests.

It is known that multicriteria methodologies help to generate knowledge about the decision context, thus, increasing the confidence of those who make decisions on the results ${ }^{5}$. This way, the judgment matrixes are constructed to obtain cardinal value scales which are implemented through the MACBETH Multicriteria Methodology ${ }^{44,49}$. The influence diagram is implemented using the GeNie tool.

Influence diagrams are now being used in decision analysis, multiple subfields of artificial intelligence, game theory, economics, stochastic modeling, etc. Applications range over the expected business problems of strategic development, R\&D, marketing, manufacturing, and capital investment, and extend to diverse areas such as breast cancer screening, gastroenterology, critical medical care, air traffic control, astronomy, and technology transfer ${ }^{6,7,8,9,10,11}$.

In this paper, we present our ongoing work on developing a hybrid model for an influence diagram based on a decision support system for multicriteria methodologies in uncertain environments. The influence diagram links all the criteria and enables us to calculate a value (within some probability distribution in the case of the uncertain criteria) for each criterion for a given action $^{12,13,14}$. This means that we can apply traditional multicriteria techniques to combine the values for a given action and then to rank the set of actions. Combining influence diagrams and multicriteria methods to provide a more complete solution for decision support under uncertainty ${ }^{15,16,17,18}$.

Applying multicriteria methods in the health area had never been considered, especially with the aim of assisting in the diagnosis of the Alzheimer's disease. The first model developed ${ }^{19}$ was validated using small study cases presented on other papers ${ }^{20}$. Then, a model $^{21,22}$ validated with a Brazilian battery ${ }^{23}$, which was based on study cases of University of São Paulo, was presented.

The model was later extended ${ }^{24}$ and validated by the data provided on the battery of CERAD ${ }^{2}$. This last 
model was based on three previous papers developed ${ }^{25}$, 26, 27

Nowadays, there are also other works which apply multicriteria methods in the health area $28,29,30,32,33,46$, 47 . The papers ${ }^{29,} 30$ present a model based mainly on a multicriteria methodology (the ZAPROS Method ${ }^{34}$ ), but with some modifications ${ }^{35}$, structured on Verbal Decision Analysis (VDA). The model aims to define which neuropathological test of CERAD would be more likely to give the faster diagnosis of the disease, based on the data of the CERAD's battery and on information given by a specialist in the health area.

Other approaches developed and based on a specific multicriteria decision aiding in classification methods to assist in the early diagnosis of Alzheimer's disease process are presented ${ }^{28,31,32}$. The multicriteria method applied is based on the concept of prototypes, being those alternatives serving as class representatives related to a given problem, and which has its performance index very dependent upon the choice of values of some control parameters. The research involves two techniques: one based on the ELECTRE IV Method, and the other, on a customized genetic algorithm. These methods are applied in order to select the prototypes and calibrate the control parameters automatically.

A hybrid proposal of an expert system integrated to a structured decision support methodology is presented $^{33}$. The methodology is also integrated to structured representations of knowledge into production rules and probabilities, which are part of the artificial intelligence area.

The battery of tests used in this work comes from the Consortium to Establish a Registry for Alzheimer's disease (CERAD). It was necessary to construct value scales originating from semantic judgments of value with the aim of defining a ranking representing the classification of the questions impact considering to the stages of the CDR.

Therefore, the paper is structured as follows: section 2 gives a brief view of how the diagnosis of the Alzheimer's disease is performed; section 3 gives an overview of the CERAD (its history, proposals, and structure), which provided the battery of tests being used in this paper; section 4 presents a step-by-step of the model structuring; and, finally, conclusions and futures works are shown in Section 5.

\section{Diagnosis of the Alzheimer's Disease}

The diagnosis of the Alzheimer's disease can be established based on several steps. This way, with the decline of the normal functioning over the nervous and other bodily systems, and with the natural behavioral and personality changes, the identification of what constitutes abnormal impairment becomes a difficult task.

The problem over the $\mathrm{AD}$ diagnosis is not only related to the current level of understanding of the disease, but also to the comprehension of the normal process involving the elderly ${ }^{36}$. For the author, there are yet no consistent set of values established for what would be a normal level of impairment in the elderly. To overcome these difficulties, some researchers $37,38,39$ have demonstrated that the $\mathrm{AD}$ first symptoms appear relatively early in life, and evolve during the lifetime. This fact raises the chances of identifying the pathology decades before a clinical diagnosis of dementia can be made.

One factor that must be considered is the brevity of the tests, that is, the shorter the test is, the more effective it will be. Among the tests recommended in order to establish the diagnosis, we can enumerate the following: for the global cognitive evaluation, the MiniMental State Examination; for the memory evaluation: delayed recall ${ }^{40}$ or the test of objects presented as drawings; for the attention: trail-making or digit-span; for the language: Boston naming; for the executive functions: verbal fluency or clock-drawing; for construction abilities: the drawing of geometric figures; and for functional evaluation, IQCODE, the Pfeffer Questionnaire or the Bayer daily activity scale ${ }^{41}$.

This study aims to help deciding which is the best manner to reach a diagnosis. To do this, we sought to choose the most important questions to the diagnosis of the Alzheimer's disease, using a battery of tests from CERAD. This battery was chosen because it encompasses all the steps of the diagnosis and it has been used all over the world.

Therefore, the questions selected through this decision making process will be applied preferentially because, in accordance to the decision maker, these questions play a main role in the diagnosis.

\section{CERAD - An Overview}

The Consortium to Establish a Registry for Alzheimer's Disease was founded in 1986 after the Health Research Extension Act of 1985 with specific focus on issues of 
diagnosis and diagnostic standardization. Its proposal was to develop a battery of standardized assessments for the evaluation of cases that had been diagnosed with Alzheimer's disease among those who had enrolled in NIA-sponsored Alzheimer's Disease Centers (ADCs) or in other dementia research program ${ }^{41}$.

At that time, regardless of the increasing interest over the illness, there was no uniform guideline over some issues such as diagnostic criteria, testing methods, and classification of the disease severity that could be followed.

This way, CERAD ${ }^{42}$ is a distinctive collaborative initiative that was created to attend to this need. As a prerequisite to the development of a national registry, a high number of universities and research centers joined their efforts to compose the CERAD.

The initial objectives of CERAD were:

(i) The standardization of the clinic data, neuropsychological, neuropathological and AD's neuroimaging evaluation;

(ii) Features identification and the analysis of the AD natural progress ${ }^{40}$.

Despite the growing interest in clinical investigations of this illness at that time, uniform guidelines were lacking in diagnostic criteria, testing procedures, and staging the illness severity. CERAD developed the following standardized instruments to assess the various manifestations of the Alzheimer's disease: Clinical Neuropsychology, Neuropathology, Behavior Rating Scale for Dementia, Family History Interviews and Assessment of Service Needs.

\section{Construction of the Model}

\subsection{Definition of Problem}

In the studies developed $24,25,26,27$, the application of the multicriteria model for aiding in the diagnosis of the Alzheimer's disease is presented. In one of the studies ${ }^{27}$, the results of the implementation of a case study conducted based on the battery of the CERAD neuropathological assessment is analyzed.

In the other ${ }^{25}$, we sought to validate the model through the neuropsychological data obtained on the patients' tests. The data used in the analysis of the study is part of the neuropsychological battery of CERAD ${ }^{41}$.

In the present study, we sought to validate the model considering the identification of issues that have greater impact on each stage of the Clinical Dementia Rating
(CDR). The diagnosis of the Alzheimer's disease will be made by the combination of the neuropsychological tests battery of CERAD based on the functional scale $\mathrm{CDR}^{36}$.

We selected six of the eight tests of the neuropsychological battery of CERAD for the application of the decision support model that will assess which issues (among all the issues that are implemented in selected tests) have the greatest attractiveness on each stage of CDR, for the definition of the diagnosis of the Alzheimer's disease. The tests selected are: Verbal Fluency (J1), Boston Naming Test (J2), Word List Memory (J4), Constructional Praxis (J5), Word List Recall (J6) and Word List Recognition (J7) ${ }^{42}$.

The CDR was chosen to be a tool that allows the classification of the prevalence of the various degrees of dementia based on six cognitive-behavioral categories: memory, orientation, judgment and problem solving, community affairs, home and hobbies, and personal care $^{42}$.

Furthermore, the CDR identifies the questionable cases, or those that are not classified as normal subjects. These cases may correspond to the so-called cognitive decline associated with aging, to the mild cognitive impairment, or to other epidemiological studies that are part of the group that has a higher rate of conversion to dementia.

Despite being represented by only five stages of dementia in the CDR (none, questionable, mild, moderate and severe), the CERAD implemented a change in the scale including two stages: profound and terminal. For the application of the model, the scale of the CDR modified by CERAD will be taken into account $^{36}$.

Next, the application of the decision model will be presented to solve the problem of choosing the questions that are considered to be the most attractive in the definition of the neuropsychological diagnosis of the Alzheimer's disease.

\subsection{Phase 1: Structuring - Identification and Organization of the Evaluation Elements}

\subsubsection{Step 1: Identifying the decision makers}

The individuals classified as cases in the database of CERAD were defined as the decision makers (actors) involved in the process of building the model to define 
the issues with the greatest impact on the neuropsychological diagnosis of AD. This decision was made considering that the degree of dementia was defined based on the values (responses) obtained in the patients tests.

Analyzing the data pertaining to the cases through the database of CERAD a negligible quantity of actors was found to evaluate the attractiveness of the model in multicriteria. The degrees of dementia none, profound and terminal are the answers 0,1 and 2 respectively, i.e. between the cases that have been assessed with the dementia-type, none, profound and terminal, only 0,1 the CERAD battery. Therefore, these degrees of dementia have not been evaluated in the model.

\subsubsection{Step 2: Identifying the alternatives and the criteria relevant to the decision issue: definition of the hierarchical structure of the problem}

This step involves the identification of the variables of interest and the determination of the interrelationships between them. The variables can be classified as: objectives, decision problem, actions, criteria, restrictions and factors, as shown in Table 1.

The end result of this step is the definition of the

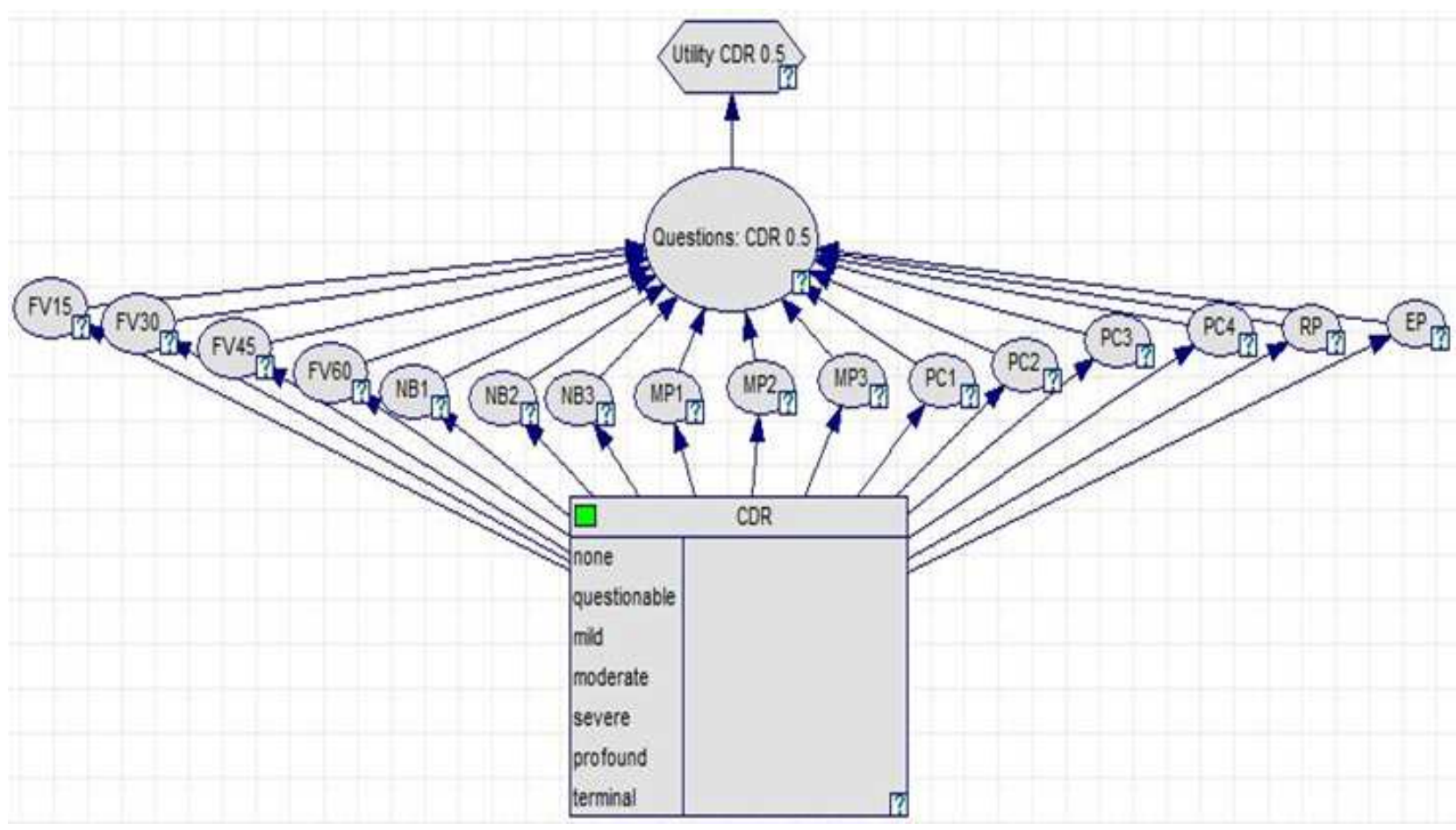

Fig. 2. Hierarchical structure of the decision problem to stage the CDR questionable.

and 2 people respectively answered each of the issues of

problem's hierarchical structure by creating a graphic

Table 1. Classification of the variables of the decision problem

Problem: Definition of the issues of greatest impact on each stage of the CDR in the neuropsychological diagnosis of the Alzheimer's disease

Objectives

The decision problem

The set of the possible actions

Criteria

Restrictions (properties of criteria that are specified as desirable)
To establish, among the various levels of dementia classified by CDR (questionable, mild, moderate, severe), which are the items that have the greatest impact on the decision of a test or set of tests for the diagnosis of the $\mathrm{AD}$

Determining which of the questions, on the neuropsychological tests of CERAD, are more attractive in each stage of CDR (questionable, mild, moderate and severe) to the definition of the diagnosis

The set of actions (alternatives) is defined as (A), where the issues are part of the neuropsychological battery of tests of the CERAD. The set selected for the implementation of the decision model is: Verbal Fluency, Boston Naming Test, Word List Memory, Constructional Praxis, Word List Recall and Word List Recognition.

Corresponds to the CDR stages of dementia that had a significant amount of actors (questionable, mild, moderate, and severe).

CDR stages

- CDR_QUESTIONABLE $>0$ and CDR_QUESTIONABLE $\leq 0.5$;

- CDR_MILD > 0.5 apdiGisiriedMUAlantis Press

- CDR_MODERATE > copdrGRRthMARAHARATE $\leq 2$;

- CDR_SEVERE > 2 and CDR_Sd $3 V E R E \leq 3$ 
model represented by a Directed Acyclic Graph (DAG), as shown in Fig. 2.

23 nodes of probability were identified, including one decision node and seven utility nodes ${ }^{41}$.

Once the network structure is defined, it is necessary to calculate the probabilities in the form of Conditional Probability Table (CTP) for each chance node of the network, based on the data obtained from the database of the CERAD.

For the definition of the most attractive issues, it is necessary to examine the level of impact (or attraction) of the actors' responses in each of the stages of the CDR. This initial assessment is important because one can discover, based on the responses of a particular descriptor for each of the fundamental points of view (FPVs), (ii) obtainment of the impacts levels according to each key point of view, and (iii) analysis of the impacts according to each fundamental point of view.

The number of states of each FPV will always be equivalent. It was defined 16 descriptors for each FPV. The states of FPVs are not equivalent; therefore, they cannot be the representation of more than one state at a single level of impact. Table 3 shows the descriptors for the FPV1. The levels of impact of each descriptor were ordered based on each issue that was relevant for the definition of each stage of the CDR, as regards the issue that has the greatest influence in defining the diagnosis of $\mathrm{AD}$. This relevance was defined based on the sum of

Table 2. Values of each level of impact for each FPV in relation to FV15

\begin{tabular}{lccccc}
\hline Alternatives & $\begin{array}{l}\text { FPV1 } \\
\text { Questionable }\end{array}$ & $\begin{array}{l}\text { CDR: } \\
\text { Mild }\end{array}$ & $\begin{array}{l}\text { FPV2 CDR: } \\
\text { Mild }\end{array}$ & $\begin{array}{l}\text { FPV3 } \\
\text { Moderate }\end{array}$ & $\begin{array}{l}\text { CDR: } \\
\text { Severe }\end{array}$ \\
\hline FV15_99 & 0.50 & & 1.00 & 2.00 & 3.00 \\
FV15_0 & 0.46 & 0.96 & 1.92 & 2.92 \\
FV15_1 & 0.42 & 0.92 & 1.84 & 2.84 \\
FV15_2 & 0.38 & 0.88 & 1.75 & 2.75 \\
FV15_3 & 0.33 & 0.84 & 1.67 & 2.67 \\
FV15_4 & 0.29 & 0.80 & 1.59 & 2.59 \\
FV15_5 & 0.25 & 0.76 & 1.50 & 2.50 \\
FV15_6 & 0.21 & 0.71 & 1.42 & 2.42 \\
FV15_7 & 0.17 & 0.67 & 1.34 & 2.34 \\
FV15_8 & 0.12 & 0.63 & 1.26 & 2.26 \\
FV15_9 & 0.08 & 0.59 & 1.18 & 2.17 \\
FV15_10 & 0.04 & 0.55 & 1.09 & 2.09 \\
FV15_11 & 0.00 & 0.51 & 1.01 & 2.01 \\
\hline
\end{tabular}

actor on the database of the CERAD, which is his/her stage of CDR.

The attractiveness of the responses is measured by means of judgment matrixes of value, and the scales of global value for each question can be obtained. Table 2 shows the value of each level of impact for all Fundamental Point of Views (FPV) in relation to FV15.

With this result, it is possible to apply the model in order to define the attractiveness of the issues involved in the neuropsychological battery of CERAD for each stage of the CDR.

\subsubsection{Step 3: Definition of descriptors}

The construction of descriptors should be made for each fundamental point of view of the problem. Thus, for this problem, two sets of descriptors have been identified considering the three phases: (i) description of each the results obtained in the judgment matrixes of decision in the application of the model on the answers of the questions.

\subsubsection{Step 4: Performing the analysis of impacts}

This step relates to the definition of the impact assessment according to each FPV. We defined the upper and lower values of each impact and the relevant aspects of the distribution of impacts in each of them. For all FPVs of this model, the scoring was attributed to the degree of dementia in accordance with each stage of the CDR which is being evaluated.

Table 4 presents the descriptors and their lower and higher values to be considered for obtaining the basis of value for each FPV. 
Table 3. Classification of variables in the decision problem

\begin{tabular}{lll}
\hline NI & Description & Order \\
\hline N16 & FV15: I want you to tell me all the animals you can think of (from 0-15 seconds) & $1^{\circ}$ \\
N15 & MP2: Repeat a list of ten words - attempt 2 & $2^{\circ}$ \\
N14 & MP3: Repeat a list of ten words - attempt 3 & $3^{\circ}$ \\
N13 & RP: Recognition of a list of ten words & $4^{\circ}$ \\
N12 & NB1: Name high frequency objects & $5^{\circ}$ \\
N11 & FV60: I want you to tell me all the animals you can think of (from 46-60 seconds) & $6^{\circ}$ \\
N10 & NB2: Name medium frequency objects & $7^{\circ}$ \\
N09 & FV30: I want you to tell me all the animals you can think of (from 16-30 seconds) & $8^{\circ}$ \\
N08 & PC2: Draw a diamond & $9^{\circ}$ \\
N07 & MP1: Repeat a list of ten words - attempt 1 & $10^{\circ}$ \\
N06 & PC3: Draw overlapping rectangles & $11^{\circ}$ \\
N05 & FV45: I want you to tell me all the animals you can think of (from 31-45 seconds) & $12^{\circ}$ \\
N04 & NB3: Name low frequency objects & $13^{\circ}$ \\
N03 & PC4: Draw a cube & $14^{\circ}$ \\
N02 & EP: Recall a list of ten words & $15^{\circ}$ \\
N01 & PC1: Draw a circle & $16^{\circ}$ \\
\hline
\end{tabular}

Table 4. Summary table of descriptors and impacts according to each FPV

\begin{tabular}{lccc}
\hline \multicolumn{1}{c}{ FPV } & Descriptor & Upper Level & Lower Level \\
\hline FPV1 - CDR: Questionable & Answers a question from the battery of & 0.51 & 0.50 \\
FPV2 - CDR: Mild & neuropsychologicy of CERAD & 1.01 & 1 \\
FPV3 - CDR: Moderate & & 2.01 & 2 \\
FPV4 - CDR: Severe & & & 3 \\
\hline
\end{tabular}

\subsection{Phase 2: Evaluation-Construction of a Quantitative Model of Values}

4.3.1. Step 5: Definition of the function of value for each alternative

This function was obtained by the division of the sum of the results obtained through the judgment matrixes in relation to the responses to a question, by the sum of the results obtained through the judgment matrixes in relation to the issue or set of issues that are part of a subtest of the neuropsychological battery of CERAD, considering a determined stage of the CDR.

$$
v\left(b_{j}\right)=\frac{\sum_{n}^{1} a_{i}}{\sum_{n}^{1} b_{j}},
$$

where: $a \in A$ (represents all the alternatives - issues), i.e. $A=\left\{a_{i}, a_{i-1}, \ldots, a_{1}\right\} ; b \in B$ (represents the subtest), i.e. $B=\left\{b_{j}, b_{j-1}, \ldots, b_{n}\right\}$ 


\subsection{Phase 4: Evaluation-Evaluation of the alternatives}

\subsubsection{Step 6: Construction of the judgment matrixes}

In this step, the following steps were performed: (i) the construction of the judgment matrixes based on the differences of attractiveness for each pair of alternatives; and (ii) the obtainment of the cardinal value scales for each fundamental point of view defined.

For the issues evaluation, all the FPVs were worked through a descriptor with 16 reference levels, and a lower limit (which was generated from the lower value, the sum obtained regarding the outcome of the evaluation of the issues), an upper limit (which was generated from the higher value, the sum obtained regarding the outcome of the evaluation of the issues) and 14 intermediate levels of reference. Fig. 3 shows a matrix of assessment of value and scale of cardinal value obtained with the methodology for the FPV1 MACBETH - CDR: Questionable ${ }^{43,44}$.

Following the procedure for construction of judgment matrixes of value and obtain the scales of global value for each of the FPV.

The result of the judgment matrixes shows that the stage of the CDR was questionable that most benefited from the implementation of the model. CDR: questionable obtained the highest value in relation to other criteria, and through the accumulated weights for each option, with the CDR: questionable accumulating $50 \%$ of the total weight of the criteria.

This result is very positive, because one of the major goals of medicine in the search for a diagnosis, especially that of Alzheimer's disease, is finding it in the earliest stages of the disease.

\begin{tabular}{|c|c|c|c|c|c|c|c|c|c|c|c|c|c|c|c|c|c|c|}
\hline \multicolumn{17}{|c|}{ Macbeth: CDR: Questionável } & \multicolumn{2}{|c|}{ () 10} \\
\hline & FY15 & MP2 & MP3 & RP & NB1 & FV60 & NB2 & $\mathrm{f} / 30$ & $\mathrm{PC2}$ & MP1 & $\mathrm{PC3}$ & FV45 & NB3 & $\mathrm{PC4}$ & EP & $\mathrm{PCI}$ & $\begin{array}{l}\text { Cunerk } \\
\text { scoste }\end{array}$ & extreme \\
\hline PV15 & no & postive & postive & very wesk & postive & postive & postive & postive & postive & vey weak & positive & vey weak & postive & postive & positive & postive & 0.50 & 1. strong \\
\hline MP2 & & no & postive & very weak & postive & very weak & postive & very weak & postive & very weak & positive & vey meak & postive & very weak & postive & very weak. & 0.47 & sliong. \\
\hline MP3 & & & no & very weak & postive & postive & postive & very weak & postive & very weak & positive & very weak & postive & postive & postive & very weak & 0.43 & notiale \\
\hline $\mathrm{RP}$ & & & & m & very weak & very weak & very weak & very weak & very weak & very weak & very weak & very weak & very weak & very weak & very weak & very weak & 0.40 & 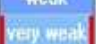 \\
\hline NB1 & & & & & m & positive & postive & very weak & possivive & very weak & postive & very weak & positive & postive & positive & very weak & 0.37 & no \\
\hline FV60 & & & & & & no & positive & postive & positive & postive & positive & positive & posstive & positive & very weak & postive & 0.33 & \\
\hline NB2 & & & & & & & no & postive & postive & very weak & positive & very weak & positive & postive & postive & postive & 0.30 & \\
\hline PV 30 & & & & & & & & no & postive & postive & positive & positive & posstive & postive & very weak & poskive & 0.27 & \\
\hline$P C 2$ & & & & & & & & & no & postive & positive & positive & postive & postive & very meak & postive & 0.23 & \\
\hline MP1 & & & & & & & & & & no & positive & postive & positive & postive & very weak & postive & 0.20 & \\
\hline $\mathrm{PC3}$ & & & & & & & & & & & no & positive & positive & postive & very weak & positive & 0.17 & \\
\hline F/45 & & & & & & & & & & & & no & positive & postive & very weak & postive & 0.13 & \\
\hline NB3 & & & & & & & & & & & & & no & postive & very weak & positive & 0.10 & \\
\hline PCA & & & & & & & & & & & & & & no & very weak. & postive & 0.07 & \\
\hline EP & & & & & & & & & & & & & & & no & very weak. & 0.03 & \\
\hline PCI & & & & & & & & & & & & & & & & no & 0.00 & \\
\hline \multicolumn{19}{|c|}{ Consistent judgements } \\
\hline 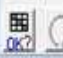 & & (8) $1+4$ & 堪 & (6) & & & & & & & & & & & & & & \\
\hline
\end{tabular}

Fig. 3. Matrix of judgment of value and scale for the FPV1 - CDR: Questionable.

\section{Conclusion}

Nowadays, great emphasis is put on identifying the cases in which the risks of developing the Alzheimer's disease are higher. As factors that contribute to this fact, we can say that there are currently few alternative therapies to the pathology treatment, and that the effectiveness of treatments is greater when an early diagnosis is possible ${ }^{36}$.

Besides, according to studies conducted by the Alzheimer's Association ${ }^{45}$, the Alzheimer's disease treatments have significant resulting costs, and it is 
known that it is one of the costliest diseases, second only to cancer and cardiovascular diseases.

The diagnosis of Alzheimer's disease can be established based on several steps. The first step is to verify whether or not the patient has any kind of dementia. In the second step, the patient is assessed in order to discover if s/he has the Alzheimer's disease.

The results of this study were obtained by the model developed through the application of methodologies such as Influence Diagrams and Multiple Criteria Decision Aid. These results reaffirm the importance of the tools to support the physicians' decision on giving the diagnosis of the Alzheimer's disease and the like, considering that it enables an accurate and differential diagnosis.

This way, a cost optimization and a reduction of the time a patient would spent without treatment (which could happen because s/he was not early diagnosed) can be provided, delaying the loss of cognitive and psychomotor abilities caused either by the disease, or other dementias.

The methodologies applied have been crucial to the analysis of the most attractive questions to the definition of the diagnosis of the Alzheimer's disease. The methodological design of the model mapped the possibilities regarding the performance results for the decision.

The model presented, which applies structured assumptions in decision-making problems, provided important impacts for the research and it was supported by the chain of neuropsychological responses to identify the diagnostic criteria.

The structure of the model applies the influence diagram, which enabled the triangulation of data for areas of cognition and functionality or praxis, based on the CERAD neuropsychological battery.

This data interface makes room for perspectives for modeling on many different areas of knowledge, whether in health, computer science, education, or others.

Therefore, the application of the influence diagrams in academic models through scientific research, aiming the costs optimization, is extremely appropriate, opening up possibilities for future studies.

\section{Future Works}

As a proposal for improvement and extension of the work, we intend to extend the model, considering the process of recommendation, so that a further analysis of the results of the model is included, such as the analysis of sensitivity and robustness.

We also intend to implement a new analysis of the model through the inclusion of values in the utility node of the influence diagram. This way, it will be possible to make inferences on the network aiming the definition of the diagnosis by the application of only the most attractive questions. Other methodologies may be applicable in the definition of the diagnosis of Alzheimer's disease ${ }^{48,49}$.

Then, an application of the model on the complete neuropsychological battery of tests of CERAD is aimed. Or even the inclusion of the forms: Mini-Mental State Examination (Mini-Mental State Examination) and Recall of Praxis (Praxis Evocation); on the set of evaluated forms.

As a final proposal, the implementation of the model to the early diagnosis of Alzheimer's disease based on batteries of tests of other countries is aimed.

\section{Acknowledgements}

The authors thank the Consortium to Establish a Registry for Alzheimer's disease (CERAD) for the divulgation of the data utilized in this case study. Isabelle Tamanini and Plácido Rogério Pinheiro are thankful to the National Counsel of Technological and Scientific Development (CNPq) for the support received on this project.

\section{References}

1. M. J. Prince, Predicting the Onset of Alzheimer's Disease Using Bayes' Theorem. Am. J. of Epidemiol. 143(3):301308, 1996.

2. G. G. Fillenbaum, G. van Belle, J. C. Morris, et al., Consortium to Establish a Registry for Alzheimer's Disease (CERAD): The first twenty years. Alzheimer's \& Dementia 4(2):96-109, 2008.

3. S. S. Bassett, Attention: Neuropsychological Predictor of Competency in Alzheimer's Disease. J. Geriatr. Psychiatr Neurology 12(4):200-205, 1999.

4. K. R. Daffner, Current Approaches to the Clinical Diagnosis of Alzheimer's Disease, in Early Diagnosis of Alzheimer's Disease, eds. L. F. M. Scinto and K. R. Daffner, 29-64, (Humana Press, New Jersey, 2000)

5. C. Evangelou, N. Karacapilidis and O. A. Khaled, Interweaving knowledge management, argumentation and decision making in a collaborative setting: the KAD ontology model. Int. J. of Knowledge and Learning 1(1/2):130-145, 2005. DOI: 10.1504/IJKL.2005.006255. 
6. R.A. Howard and J.E. Matheson, Influence Diagrams. Decision Analysis, 2(3):127-143, 2005

7. R.A. Howard and J.E. Matheson, Influence Diagram Retrospective. Decision Analysis, 2(3):144-147, 2005

8. A. Detwarasiti and R.D. Shachter, Influence Diagrams for Team Decision Analysis. Decision Analysis, 2(4): 207-228, 2005.

9. C. Boutilier, The Influence of Influence Diagrams on Artificial Intelligence. Decision Analysis, 2(4):229-231, 2005.

10. D.M. Buede, Influence Diagrams: A Practitioner's Perspective. Decision Analysis, 2(4):235-237, 2005

11. S.G. Pauker and J.B. Wong, The Influence of Influence Diagrams in Medicine. Decision Analysis, 2(4):238-244, 2005.

12. J.Meyer, M.H. Phillips, P.S. Cho, I. Kalet and J.N. Doctor. Application of influence diagrams to prostate intensity-modulated radiation therapy plan selection. Institute of Physics Publishing Physics in Medicine and Biology. Phys. Med. Biol., 49, 1637-1653, 2004.

13. D.T. Maxwell and D.M. Buede. Composing and Constructing Value Focused Influence Diagrams: A Specification for Decision Model Formulation. Journal of Multi-Criteria Decision Analysis. 12(4-5): 225-243, 2003

14. I. Linkov, S. Sahay, G. Kiker, T. Bridges and T.P. Seager. Multi-Criteria Decision Analysis: A Framework for Managing Contaminated Sediments. Comparative Risk Assessment and Environmental Decision Making, (2004) 15-54.

15. W. Watthayu. Representing and Solving Influence Diagram in Multi-Criteria Decision Making:

16. A Loopy Belief Propagation Method, in: Proceedings of the International Symposium on Computer Science and its Applications (CSA 2008), 118-125 (Hobart, Australia, 2008).

17. W. Watthayu, Y. Peng. A Bayesian network based framework for multi-criteria decision making, in: Proceedings of the 17th International Conference on Multiple Criteria Decision Analysis (MCDM), 6-11 (Whistler, Canada, 2004).

18. N. Fenton and M. Neil. Making Decisions: Using Bayesian Nets and MCDA. Knowledge-Based Systems, 14:307-325,2000

19. Ana Karoline A. Castro, Plácido R. Pinheiro, Mirian Caliope D. Pinheiro, Applying a Decision Making Model in the Early Diagnosis of Alzheimer's Disease, in: Proc. Second International Conference on Rough Set and Knowledge Technology (RSKT 2007), eds. J. Yao, P. Lingras, W. -Z. Wu, M. Szczuka, N. Cercone and D. Slezak. Lecture Notes in Computer Science, 4481:149156, (Toronto, Canada 2007).

20. J. Morris, The Clinical Dementia Rating (CDR): Current Version and Scoring Rules, Neurology 43(11):24122414,1993

21. Ana Karoline A. Castro, Plácido R. Pinheiro and Mirian Caliope D. Pinheiro, A Multicriteria Model Applied in the Diagnosis of Alzheimer's Disease, in: Third
International Conference on Rough Set and Knowledge Technology (RSKT 2008), eds. G. Wang, T. Li, J. W. Grzymsls-Busse, D. Miao, A. Skowron and Y. Y. Yao. Lecture Notes in Computer Science, 5009:612-619 (Chengdu, China, 2008).

22. Ana Karoline A. Castro, P. R. Pinheiro and M. C. D. Pinheiro, A Multicriteria Model Applied in The Early Diagnosis of Alzheimer's: A Bayesian Approach, in: Proc. VI International Conference on Operational Research for Development 6:9-19, (Fortaleza, Brazil, 2007)

23. A.C.S. Silva, Métodos Quantitativos Aplicados a Políticas de Saúde Pública: Estudo de caso dos idosos. Master Thesis - Instituto Tecnológico de Aeronáutica (ITA), São José dos Campos, 2006

24. Ana Karoline A. Castro, Plácido R. Pinheiro and Mirian Caliope D. Pinheiro, An Approach for the Neuropsychological Diagnosis of Alzheimer's Disease, in: Fourth International Conference on Rough Set and Knowledge Technology (RSKT), eds. G. Wang, T. Li, J. W. Grzymsls-Busse, D. Miao, A. Skowron and Y. Y. Yao. Lecture Notes in Computer Science, 5589:216-223, (Gold Coast, Australia, 2009).

25. Ana Karoline A. Castro, Placido R. Pinheiro and Mirian Caliope D. Pinheiro, A Hybrid Model for Aiding in Decision Making for the Neuropsychological Diagnosis of Alzheimer's Disease, in: Proc. 6th International Conference Rough Sets on and Current Trends in Computing (RSCTC), eds. C. -C. Chan, J. W. GrzymalaBusse and W. P. Ziarko. Lecture Notes in Computer Science, 5306:495-504, (Akron, Ohio, 2008)

26. Ana Karoline A. Castro, Um Modelo Híbrido Aplicado ao Diagnóstico da Doença de Alzheimer. Master Thesis Master Program in Applied Computer Sciences, University of Fortaleza (2009)

27. Plácido R. Pinheiro, Ana Karoline A. Castro and Mirian Caliope D. Pinheiro, Multicriteria Model Applied in the Diagnosis of Alzheimer's Disease: A Bayesian Network, in Proceedings of the 11th IEEE International Conference on Computational Science and Engineering, 15-22, (São Paulo, Brazil, 2008).

28. Amaury T. Brasil, Plácido R. Pinheiro, Andre L. V. Coelho and N. C. Costa, Comparison of Two MCDA Classification Methods over the Diagnosis of Alzheimer's Disease, in Proc. Fourth International Conference on Rough Set and Knowledge Technology (RSKT), eds. G. Wang, T. Li, J. W. Grzymsls-Busse, D. Miao, A. Skowron and Y. Y. Yao. Lecture Notes in Computer Science, 5589: 334-341 (Gold Coast, Australia, 2009) DOI: 10.1007/978-3-642-02962-2_42

29. Isabelle Tamanini, Ana Karoline A. Castro, Plácido R. Pinheiro and Mirian Caliope D. Pinheiro, Towards the early diagnosis of Alzheimer disease: A multicriteria model structured on neuroimaging. Int. J. of Social and Humanistic Computing, 1:203-217, 2009

30. Isabelle Tamanini, Ana Karoline A. Castro, Plácido R. Pinheiro and Mirian Caliope D. Pinheiro, Towards an 
Applied Multicriteria Model to the Diagnosis of Alzheimer's Disease: A Neuroimaging Study Case, in 2009 IEEE International Conference on Intelligent Computing and Intelligent Systems (ICIS), 652-656, 2009

31. Amaury T. Brasil, A Novel Approach Based on Multiple Criteria Decision Aiding Methods to Cope with Classification Problems. Master Thesis - Master Program in Applied Computer Sciences, University of Fortaleza (2009)

32. Amaury T. Brasil, Plácido R. Pinheiro, Andre L. V. Coelho and N. C. Costa, Towards the Early Diagnosis of Alzheimer's Disease via a Multicriteria Classification Model, in 5th Evolutionary Multi-Criterion Optimization (EMO 2009). Lecture Notes in Computer Science, 5467: 393-406, (Nantes, France, 2009) DOI: 10.1007/978-3642-01020-0_32.

33. Luciano C. Nunes, Plácido R. Pinheiro and Tarcisio C. Pequeno, An Expert System Applied to the Diagnosis of Psychological Disorders, IEEE International Conference on Intelligent Computing and Intelligent Systems (ICIS), 363-367, 2009.

34. O. Larichev, Ranking Multicriteria Alternatives: The Method ZAPROS III. Eur. J. Op. Res. 131(3) (2001) 550-558.

35. Isabelle Tamanini and Plácido R. Pinheiro, Challenging the Incomparability Problem: An Approach Methodology Based on ZAPROS. Modeling, Computation and Optimization in Information Systems and Management Sciences, Communications in Computer and Information Science, Springer-Heidelberg 14(1):344-353,2008, DOI: 10.1007/978-3-540-87477-5_37

36. A. D. Davidoff, Issues in the clinical diagnosis of Alzheimer's disease. Am. J. of Alzheimer's Disease and Other Dementias 1(1):9-15, 1986

37. C. H. Kawas, M. M. Corrada, R. Brookmeyer, A. Morrison, S. M. Resnick, A. B. Zonderman and D. Arenberg, Visual memory predicts Alzheimer's disease more than a decade before diagnosis. Neurology, $\mathbf{6 0}(7)$ : 1089-1093, 2003

38. H. Braak and E. Braak, Frequency of stages of Alzheimer-related lesions in different age categories. Neurobiol Aging, 18(4):351-357, 1997.

39. M. F. Elias, A. Beiser, P. Wolf, R. Au, R. F. White and R. B. D'Agostino, The preclinical phase of Alzheimer disease: A 22-year prospective study of the Framinghan cohort. Arch. Neurol. 57(6):808-813, 2000.

40. E. Mohr, D. Dastoor and J. J. Claus, Neuropsychological Assessment. In: Clinical Diagnosis and Management of Alzheimer's Disease, ed. S. Gauthier, 93-106, (Martin Dunitz, London, 1999)

41. Genie. Graphical Network Interface. Decision Systems Laboratory, University of Pittsburgh, 2008.

42. J. C. Morris, A. Heyman, R. C. Mohs, et al.: The Consortium to Establish a Registry for Alzheimer's Disease (CERAD): Part 1. Clinical and Neuropsychological Assessment of Alzheimer's Disease. Neurology 39(9):1159-1165, 1989
43. C. A. Bana e Costa, J. C. Vansnick., The MACBETH approach: Basic ideas, software, and an application, International Conference on Methods and Applications of Multiple Criteria Decision Making, 4:131-157, 1999.

44. C. A. Bana e Costa, M.P. Chagas, A career choice problem: An example of how to use MACBETH to build a quantitative value model based on qualitative value judgments, European Journal of Operations Research, 153(2):323-331, 2004.

45. R. Koppel, Alzheimer's Disease: The Costs to U.S. Businesses in 2002. Alzheimers Association - Report (2002).

46. Isabelle Tamanini, Plácido R. Pinheiro, Mirian Caliope D. The Neuropathological Diagnosis of the Alzheimer's Disease under the Consideration of Verbal Decision Analysis Methods. Fifth International Conference on Rough Set and Knowledge Technology (RSKT), 2010. (accepted for publication)

47. Isabelle Tamanini, Plácido R. Pinheiro, Mirian Caliope D. Pinheiro, The Choice of Neuropathological Questionnaires to Diagnose the Alzheimer's Disease Based on Verbal Decision Analysis Methods. International Conference on Information Computing and Applications (ICICA), Lecture Notes in Computer Science 6377:549-566, 2010

48. Gürbüz, T., Multiple Criteria Human Performace Evaluation Using Choquet Integral. International Journal of Computational Intelligence Systems ( IJCIS), 3(3): 290-300, 2010

49. Cengiz Kahraman, A. Cagri Tolga, An Alternative Ranking Approach and Its Usage in Multi-Criteria Decision-Making. International Journal of Computational Intelligence Systems ( IJCIS), 2(3):219-235, 2009 